\title{
Impacts of Nonsynonymous Single Nucleotide Polymorphisms of Adiponectin Receptor 1 Gene on Corresponding Protein Stability: A Computational Approach
}

\author{
Md. Abu Saleh, ${ }^{1}$ Md. Solayman, ${ }^{1}$ Sudip Paul, ${ }^{1}$ Moumoni Saha, ${ }^{1}$ \\ Md. Ibrahim Khalil, ${ }^{1,2}$ and Siew Hua Gan ${ }^{2}$ \\ ${ }^{1}$ Department of Biochemistry and Molecular Biology, Jahangirnagar University, Savar, Dhaka 1342, Bangladesh \\ ${ }^{2}$ Human Genome Centre, School of Medical Sciences, Universiti Sains Malaysia, 16150 Kubang Kerian, Kelantan, Malaysia \\ Correspondence should be addressed to Sudip Paul; sudippaul.bcmb@gmail.com
}

Received 15 February 2016; Accepted 11 April 2016

Academic Editor: Ryuji Hamamoto

Copyright (C) $2016 \mathrm{Md}$. Abu Saleh et al. This is an open access article distributed under the Creative Commons Attribution License, which permits unrestricted use, distribution, and reproduction in any medium, provided the original work is properly cited.

Despite the reported association of adiponectin receptor 1 (ADIPOR1) gene mutations with vulnerability to several human metabolic diseases, there is lack of computational analysis on the functional and structural impacts of single nucleotide polymorphisms (SNPs) of the human ADIPOR1 at protein level. Therefore, sequence- and structure-based computational tools were employed in this study to functionally and structurally characterize the coding nSSNPs of ADIPOR1 gene listed in the dbSNP database. Our in silico analysis by SIFT, nsSNPAnalyzer, PolyPhen-2, Fathmm, I-Mutant 2.0, SNPs\&GO, PhD-SNP, PANTHER, and SNPeffect tools identified the nsSNPs with distorting functional impacts, namely, rs765425383 (A348G), rs752071352 (H341Y), rs759555652 (R324L), rs200326086 (L224F), and rs766267373 (L143P) from 74 nsSNPs of ADIPOR1 gene. Finally the aforementioned five deleterious nsSNPs were introduced using Swiss-PDB Viewer package within the X-ray crystal structure of ADIPOR1 protein, and changes in free energy for these mutations were computed. Although increased free energy was observed for all the mutants, the nsSNP H341Y caused the highest energy increase amongst all. RMSD and TM scores predicted that mutants were structurally similar to wild type protein. Our analyses suggested that the aforementioned variants especially H341Y could directly or indirectly destabilize the amino acid interactions and hydrogen bonding networks of ADIPOR1.

\section{Introduction}

In recent years, the number of obese individuals has been dramatically increased throughout the world which leads to the acceleration of obesity related health problems $[1,2]$. Decreased insulin sensitivity, the most common arena of obesity, predisposes the affected persons to a variety of pathological abnormalities including type 2 diabetes, hypertension, and cardiovascular diseases [3-5]. The concomitance of these diseases has been considered as metabolic syndrome. In multiple studies, it has been reported that genetic variations in the adiponectin gene are associated with these types of diseases [6]. Adiponectin is an adipokine or adipocytokine specially secreted by adipocytes [7] and placenta during pregnancy [8] that circulates at relatively high $(2-20 \mathrm{mg} / \mathrm{mL})$ concentrations in the blood stream. Biologically active adiponectin hormone is a collagen-like circulating protein which acts as a principle antidiabetic and antiatherogenic adipokine [9-12]. Reduced adiponectin level in plasma has been observed in obesity, insulin resistance, and type 2 diabetes [9-12]. Adiponectin exerts its insulin sensitizing effects by increasing fatty-acid oxidation via activation of AMP-activated protein kinase (AMPK) peroxisome proliferator-activated receptor-alpha $(\operatorname{PPAR}-\alpha)[13,14]$. Therefore, adiponectin is anticipated to be a novel therapeutic target for diabetes and the metabolic syndrome.

To employ proper functions, adiponectin binds to a number of receptors. Different studies have identified two receptors named adiponectin receptor-1 (ADIPOR1) and adiponectin receptor-2 (ADIPOR2) (those are homologous to $G$ protein-coupled receptors) as well as one receptor similar to the cadherin family $[15,16]$. In human beings, 
ADIPOR1 and ADIPOR2 genes are located at chromosomal locations 1p36.13-q41 and 12p13.31, respectively [17]. The expression of ADIPOR1 gene is found principally in skeletal muscles but may be presented ubiquitously also, while the expression of ADIPOR2 is the most abundant in liver [17]. Among these receptors, ADIPOR1 plays crucial roles in regulation of energy homeostasis as well as glucose and lipid metabolism [18]. According to several studies conducted, single nucleotide polymorphisms (SNPs) in ADIPOR1 gene can hamper the physiological functions exerted by the ADIPOR1 protein. A recent comprehensive investigation on a European-Australian population has established the association of genetic variation in adiponectin receptors with type 2 diabetes $[19,20]$. Moreover, some SNPs of ADIPOR1 gene have been found to exert significant effects on the risk of prostate cancer (rs12733285) [21], insulin resistance (rs1342387) [22], and even liver fat deposition (-1927 T/C) [23]. Although there are several in vivo studies describing the association of SNPs in the ADIPOR1 gene with metabolic disorders $[24,25]$, computational analysis has not yet been undertaken on the functional and structural consequences of nsSNPs in this gene.

In current years, computational tools are being widely used to characterize the impacts of deleterious nsSNPs in candidate genes by utilizing the information obtained from physicochemical properties of polypeptides [26, 27], conserved sequences across the species [28], and their structural attributes [29]. With the help of computational algorithms, several in silico studies have effectively filtrated functional SNPs out of large pool of diseases sensitive SNPs of BRCA1, ATM [30], and PON1 [31] genes based on their functional consequences and structural stabilities. In spite of the availability of undoubted data referring the extensive involvement of ADIPOR1 gene mutations in human diseases, the computational analysis of nsSNPs is still unveiled.

In this study, the clinical variants of ADIPOR1 were collected for in silico analysis. By utilizing these data, we employed different publicly available bioinformatics tools and databases for a comprehensive analysis of nsSNPs in ADIPOR1 gene. We also calculated the free energy changes for mutants and wild type ADIPOR1 protein in order to evaluate their stability. This study might be helpful for further investigation in order to discover new therapeutic drugs related to adiponectin receptor 1 associated diseases.

\section{Methods and Materials}

2.1. SNP Data Mining. The data on the human ADIPOR1 gene were obtained from web-based data sources such as Online Mendelian Inheritance in Man (OMIM; http://www.ncbi .nlm.nih.gov/omim/) and the National Center for Biological Information (http://www.ncbi.nlm.nih.gov/). The information about SNPs of ADIPOR1 gene of Homo sapiens was collected from the dbSNP-NCBI (http://www.ncbi.nlm.nih.gov/ SNP/) [32] for further computational analysis. The protein sequence of $A D I P O R 1$ gene was obtained from UniProtKB database (http://www.uniprot.org/uniprot/).
2.2. Analysis of the Functional Consequence of nsSNPs by Sorting Intolerant from Tolerant (SIFT). SIFT (http://sift.jcvi.org/) predicts the deleterious and tolerated SNPs in order to characterize the consequences of amino acid substitutions on phenotypic and functional changes of protein molecules. By using sequence homology based method, SIFT assumes that significant positions in a protein sequence have been conserved throughout evolution and, therefore, substitutions at these positions may affect protein function. The identification numbers (rsIDs) of each nsSNP of ADIPOR1 gene were submitted as an input to SIFT server for homology searching. SIFT calculates the SIFT score or tolerance index (TI) score for each nsSNP. The SIFT value $\leq 0.05$ indicates the deleterious effect of nonsynonymous variants on protein function [33].

2.3. Investigation of Functional Impacts of nsSNPs by nsSNPAnalyzer. nsSNPAnalyzer (http://snpanalyzer.uthsc.edu/) server was used to predict whether a nsSNP of ADIPOR1 protein affects its phenotypic effect. The input options for nsSNPAnalyzer are protein sequences in FASTA format and detailed information on amino acid substitutions. This server usually uses information contained in the multiple sequence alignment and the 3D structure in order to make a prediction. The prediction of this tool is based on a machine learning method known as Random Forest. The results of this server depict whether an nsSNP is associated with disease or neutral [34].

2.4. Analysis of the Functional Impacts of nsSNPs by Screening for Nonacceptable Polymorphisms (SNAP2). To find the functional effects of nsSNP, SNAP2 (https://rostlab.org/services/ snap $2 w e b /$ ) server was used. The prediction done by SNAP2 is based on a learning device method known as neural network. In order to make a prediction, SNAP2 utilizes the information of automatically created multiple sequence alignment and also some structural features such as predicted secondary structure and solvent accessibility. FASTA format of protein sequences is only the input option for SNAP2. The output of this server consists of prediction (Effect or neutral), score (ranges from -100 strong neutral prediction to +100 strong effect prediction), and expected accuracy [35].

2.5. Characterization of Functional Consequence of nsSNPs by PolyPhen-2. PolyPhen-2 (http://genetics.bwh.harvard.edu/ pph2/) is an advanced version of the PolyPhen tool that was used to find out the possible effect of an amino acid substitution on the structure and function of ADIPOR1 protein. UniProtKB accession number/FASTA sequence and details of amino acid substitutions are required for the input options of PolyPhen-2 server. This tool calculates Naïve Bayes posterior probability that this mutation is damaging and reports estimation of corresponding false positive and true positive rate. A mutation is estimated qualitatively as probably damaging (probabilistic score $>0.85$ ), possibly damaging (probabilistic score $>0.15$ ), and benign (remaining) with specificity and sensitivity values [36]. 
2.6. Prediction of Disease Related nsSNPs by SNPsঊGO. SNPs\&GO (http://snps.biofold.org/snps-and-go/snps-andgo.html) is a support vector machine (SVM) based classifier [37]. This server accurately predicts the mutation related to disease from protein sequence. The probability score greater than 0.5 indicates that the disease related effect is caused by nsSNPs on the function of parent protein. The whole protein sequence in FASTA format is the input for this server. The server also provides the output display for additional two servers such as PHD-SNP [38] and PANTHER [39] algorithms.

2.7. Functional Analysis of nsSNP through Hidden Markov Models (Fathmm). Fathmm (http://fathmm.biocompute.org .uk/inherited.html) not only predicts the potentially deleterious nature of protein variants but also the skill of annotating the molecular and phenotypic consequences of these mutations [40]. This server is composed of two algorithms: sequence/conservation based (unweighted) and other combined sequence conservation with pathogenicity weights (weighted). In this study, we used weighted algorithm because this algorithm is capable of adjusting conservation-based predictions to account for the tolerance of related sequences to mutations.

2.8. Investigation of the Molecular Phenotypic Effects of nsSNPs by SNPeffect. The SNPeffect database 4.0 (http://snpeffect .switchlab.org/) utilizes sequence- and structure-based bioinformatics tools in order to make prediction of molecular phenotypic impacts of nsSNP on ADIPOR1 gene. This server mainly integrates three different tools such as TANGO, WALTZ, and LIMBO and also uses FoldX server to find out a decision whether the mutation is stabilizing or destabilizing the structure of native proteins. TANGO algorithm identifies the aggregation prone regions in a protein sequence by calculating the hydrophobicity and beta-sheet forming propensity. WALTZ algorithm predicts amyloid forming regions in protein sequences with accuracy and specificity, while LIMBO algorithm predicts a chaperone binding site for the Hsp70 chaperones. The input options are usually composed of FASTA sequence/PDB ID/PDB file/UniProt ID and details of nsSNP [41].

2.9. Prediction of Protein Stability Changes upon nsSNPs by I-Mutant 2.0. I-Mutant 2.0 (http://folding.biofold.org/imutant/i-mutant2.0.html) is a support vector machine(SVM-) based tool which was used to predict the protein stability changes upon nsSNPs. In this study, sequence of protein, temperature $\left(25^{\circ} \mathrm{C}\right), \mathrm{pH}(7)$, and details of nsSNPs were used as input parameters to this server. The output is a free energy change value $(\Delta \Delta \mathrm{G})$ of protein after and before mutation. Positive $\Delta \Delta G$ value concludes that the protein being mutated is of higher stability and vice versa is also true [42].

2.10. Identification of Functional Regions in Proteins by ConSurf. ConSurf (http://consurf.tau.ac.il/) is a web-based tool that automatically analyzes evolutionary conservation of amino acid substitutions in protein by using an empirical
Bayesian inference. This server is composed of combining two self-governing servers (ConSeq and ConSurf). After providing the FASTA sequence of ADIPOR1 protein to ConSurf tool, the conserved regions were predicted with conservation grades color-coded onto its surface that can finally be pictured online using the Protein Explorer engine [43].

2.11. Analysis of Impacts of nsSNPs on Surface and Solvent Accessibility of Protein by NetSurfP. The active site of a protein in its three-dimensional conformation can be traced by surface and solvent accessibility region of amino acids of that protein. The FASTA sequence of ADIPOR1 protein was submitted to NetSurfP (http://www.cbs.dtu.dk/services/ NetSurfP/) server in order to predict its secondary structure, surface, and solvent accessibility of amino acids [44]. The output of this server provides 3 subclasses defined for solvent accessibility of amino acids: low accessibility (buried), moderate accessibility (partially buried), and high accessibility (exposed).

2.12. Modeling the Molecular Effects of nsSNPs on Protein Structure and Evaluating Their Difference of RMSD Value and TM Score. Structural analysis was done in order to explore the structural deviations and stability differences between native and mutant forms of ADIPOR1 proteins. The crystal structure of ADIPOR1 protein available in Protein Data Bank (PDB) [45] has an ID 3WXV. The ADIPOR1 protein contains 375 amino acids from which 287 amino acids have been resolved in crystal structure with a resolution of $2.90 \AA$ [46]. The Swiss-PDB viewer [47] was utilized in order to carry out amino acid substitutions, followed by the energy minimization of the modeled 3D structure of variants using a version of the GROMOS 43B1 force field in GROMOS96 software package embraced in the Swiss-PDB viewer. TMAlign was used to calculate the TM scores and root mean square deviations (RMSDs) [48].

2.13. Identification of Ligand Binding Sites on Unbound Protein Structure by FTSite. Detection of ligand binding sites on unbound proteins is essential to elucidate the protein structure-function relation and for protein engineering. FTSite (http://ftsite.bu.edu/) predicts ligands or small molecule binding sites of proteins based on experimental evidence with $94 \%$ accuracy [49]. The input options of this server generally consist of job name, Protein Data Bank ID (PDB ID) or file, and also PDB chain ID if proteins contain multiple subunits.

2.14. Investigation of Protein-Protein Interactions. Proteinprotein interaction networks are important to investigate the functions of the interactions of a particular protein with other proteins at cellular level. Online database resource Search Tool for the Retrieval of Interacting Genes (STRING) was applied to identify the interactions of ADIPOR1 protein with other corresponding proteins [50]. This server provided a unique coverage and ease of access to both experimental and predicted interaction information of ADIPOR1. In this study, 
we operated KEGG (http://www.genome.jp/kegg/) PATHWAY and LIGAND to make prediction of the functional networking of ADIPOR1 protein.

\section{Results and Discussion}

3.1. Retrieval of SNPs. The dbSNP-NCBI database was searched for retrieving the SNPs in the human ADIPOR1 gene (Gene ID: 51094). A total of 138 SNPs were found in the exonic region, among them $62(44.93 \%)$ were synonymous, 74 (53.62\%) nonsynonymous and missense, 1 (0.72\%) nonsynonymous and nonsense, and $1(0.72 \%)$ frame-shift mutations. However, only nonsynonymous SNPs were selected from coding region for this computational analysis.

3.2. Detection of Functional nsSNPs in Exonic Regions. The searching of functionally significant nsSNPs was done by predicting those which substitute the amino acids that are critical for ADIPOR1 gene function. This computational study was accomplished and authenticated using different in silico tools, namely, SIFT, nsSNPAnalyzer, SNAP2, PolyPhen-2, SNPs\&GO, Fathmm, SNPeffect, and I-Mutant 2.0.

3.2.1. Analysis of Phenotypic Impacts by SIFT. SIFT tools filtrated that a total of 13 variants $(17.568 \%)$ were damaging (score of $0.00-0.04$ ) and the remaining 61 variants $(82.432 \%$ ) became tolerated (score of $0.08-0.55$ ). It was noted that, among 13 variants, 2 nsSNPs (rs765487840, rs775780092) were predicted as damaging with low confidence. Therefore, SIFT suggested that these 11 nsSNPs might disrupt both the protein function and structure. The detailed results are provided in supporting information (see Table S1 of the Supplementary Material available online at http://dx.doi.org/ $10.1155 / 2016 / 9142190)$.

3.2.2. Functionally Significant nsSNPs by nsSNPAnalyzer and SNAP2. The results obtained from the nsSNPAnalyzer (Table S2) predicted that a total of 27 nsSNPs (36.486\%) might be disease causal. In contrast, 47 nsSNPs (63.513\%) have no effect on protein function and, hence, are considered as neutral. In addition, the results from SNAP2 server (Table S2) indicated 19 variants $(25.675 \%)$ as significant and the remaining nsSNPs (74.324\%) as neutral. Among the three computational tools, the highest number of significant nsSNP (27 variants) was detected by the nsSNPAnalyzer. The results obtained from SIFT, nsSNPAnalyzer, and SNAP2 concluded that the 7 nsSNPs with rsIDs of rs764078304, rs765425383, rs752071352, rs759555652, rs764912508, rs200326086, and rs766267373 are found as significant among three servers and thereby the result has one step refined and validated (Table 1).

3.2.3. Simulation of Functional Consequences by PolyPhen2. The results (Table S3) obtained from PolyPhen-2 server indicated that $12(16.216 \%)$ out of 74 nsSNPs were predicted as probably damaging (score of 0.96-1.00; more confident prediction) and 12 (16.216\%; less confident prediction) nsSNPs were ranked as possibly damaging (score of 0.531-0.874) as well. Meanwhile, 50 (67.567\%) nsSNPs were also classified as benign (score of 0.411-0.000). The classification of SNPs on the basis of PolyPhen-2 scores permits us to assess the potential quantitative effect of SNPs on wild type protein. Moreover, 7 nsSNPs (rs765425383, rs752071352, rs759555652, rs200326086, rs772408783, rs766267373, and rs749789403), predicted as damaging by SIFT, are also found as damaging using PolyPhen-2. This result gives clear indication that there is a strong correlation exists between evolutionary based approaches SIFT and the structural based approach PolyPhen-2 tools.

\subsubsection{Functional Characterization by PhD-SNP, PANTHER,} SNPseGO, and Fathmm. We performed PhD-SNP, PANTHER, SNPs\&GO, and Fathmm analyses of human ADIPOR1 nsSNPs in order to add another layer of refinement in nsSNPs characterization. The predicted results by these servers are shown in Tables S4 and S5.

The predictions gained form PhD-SNP server offer the fact that 29 nsSNPs cause disease with probability score greater than 0.5 and the remaining nsSNPs are marked as neutral. The number of disease causing variants has been decreased in case of the prediction of PANTHER and SNPs\&GO tools. The disease causing variants predicted by PANTHER and SNPs\&GO are 15 and 13 nsSNPs, respectively. The results from PANTHER server showed that 17 nsSNPs remain unclassified.

From Fathmm, the nsSNPs in amino acids positions 4 to 122 in human ADIPOR1 protein are found to be damaging with score of -3.97 to -4.20 .

The efficacy of functional SNP prediction can be increased more reliably by integrating the results of SVM based approaches. By combining the predictions of SIFT, nsSNPAnalyzer, SNAP2, PolyPhen-2, PhD-SNP, PANTHER, SNPs\&GO, and Fathmm, five nsSNPs (A348G, H341Y, R324L, L224F, and L143P) are found to br more deleterious and disease associated (Tables 1 and 2).

3.2.5. Functional Investigation by SNPeffect. Biological macromolecules including proteins undergo self-assembly into functional complex in a tightly regulated manner to conduct the defined function [51]. Failure of correct aggregation of proteins may result in some conditions including type 2 diabetes, Alzheimer's disease, and other neurological diseases [52]. The results from TANGO investigation presented that only two variants, namely, A348G (dTANGO score is -39.32) and R324L (dTANGO score is -1.01), were found to be not affected among 5 selected variants in the aggregation prone regions of ADIPOR1 protein. In addition, the aggregation tendency of the other two variants, H341Y (dTANGO score is 222.09) and L224F (dTANGO score is 51.62), was increased and only one variant (L143P) with dTANGO score of -230.61 was decreased. On the other hand, WALTZ analysis screened that H341Y mutant (dWALTZ score -241.53) was found to be decreased to protein amyloid forming propensity and the rest of mutants were not affected. LIMBO prediction revealed that no variants were detected to modify the chaperone binding sites for Hsp70 chaperones. In this study, we analyzed the variants by SNPeffect tools at $90 \%$ homology searching of protein structures. SNPeffect could 
TABLE 1: Refined SNPs obtained from SIFT, SNAP2, and nsSNPAnalyzer based classifications system.

\begin{tabular}{|c|c|c|c|c|c|c|}
\hline \multirow{2}{*}{ rsIDs } & \multirow{2}{*}{ Amino acid change } & \multicolumn{2}{|c|}{ SIFT } & \multicolumn{2}{|c|}{ SNAP2 } & \multirow{2}{*}{$\begin{array}{c}\text { nsSNPAnalyzer } \\
\text { Prediction }\end{array}$} \\
\hline & & Score & Prediction & Score & Prediction & \\
\hline rs764078304 & G367R & 0.04 & Damaging & 72 & Effect & Disease \\
\hline rs139371614 & G364S & 0.79 & Tolerated & -74 & Neutral & Disease \\
\hline rs765425383 & A348G & 0 & Damaging & 34 & Effect & Disease \\
\hline rs752071352 & $H 341 Y$ & 0 & Damaging & 80 & Effect & Disease \\
\hline rs759555652 & $R 324 L$ & 0.02 & Damaging & 56 & Effect & Disease \\
\hline rs778848411 & V279G & 0.02 & Damaging & 36 & Effect & Neutral \\
\hline rs759593783 & $\mathrm{G} 275 \mathrm{~A}$ & 0.24 & Tolerated & 45 & Effect & Disease \\
\hline rs764912508 & $R 264 W$ & 0.02 & Damaging & 49 & Effect & Disease \\
\hline rs369530077 & $\mathrm{I} 251 \mathrm{~N}$ & 0.01 & Damaging & 19 & Effect & Neutral \\
\hline rs200326086 & $L 224 F$ & 0.01 & Damaging & 2 & Effect & Disease \\
\hline rs772408783 & L215V & 0.02 & Damaging & 1 & Effect & Neutral \\
\hline rs756988796 & R202W & 0.08 & Tolerated & -35 & Neutral & Disease \\
\hline rs772165061 & V200L & 0.23 & Tolerated & 8 & Effect & Neutral \\
\hline rs760115326 & F173L & 0.1 & Tolerated & 48 & Effect & Disease \\
\hline rs770463342 & K170N & 0.09 & Tolerated & 25 & Effect & Neutral \\
\hline rs767286210 & L149F & 0.32 & Tolerated & -50 & Neutral & Disease \\
\hline rs780018580 & F145L & 0.75 & Tolerated & -45 & Neutral & Disease \\
\hline rs766267373 & L143P & 0.01 & Damaging & 75 & Effect & Disease \\
\hline rs764226232 & R122W & 0.17 & Tolerated & -36 & Neutral & Disease \\
\hline rs751626519 & M118K & 0.2 & Tolerated & 60 & Effect & Disease \\
\hline rs781585434 & P116S & 0.18 & Tolerated & 6 & Effect & Neutral \\
\hline rs749789403 & D108N & 0.02 & Damaging & -10 & Neutral & Neutral \\
\hline rs141511034 & P96L & 0.33 & Tolerated & -75 & Neutral & Disease \\
\hline rs769729230 & E78K & 0.15 & Tolerated & 31 & Effect & Disease \\
\hline rs751028180 & G31E & 1 & Tolerated & -81 & Neutral & Disease \\
\hline rs149582032 & $\mathrm{A} 28 \mathrm{~T}$ & 0.61 & Tolerated & -88 & Neutral & Disease \\
\hline rs780838176 & E26Q & 0.34 & Tolerated & -89 & Neutral & Disease \\
\hline rs749145406 & A15P & 0.35 & Tolerated & -34 & Neutral & Disease \\
\hline rs200868442 & G14F & 0.91 & Tolerated & -4 & Neutral & Disease \\
\hline rs774465119 & $\mathrm{N} 13 \mathrm{~K}$ & 0.93 & Tolerated & 18 & Effect & Disease \\
\hline rs372656012 & G12E & 1 & Tolerated & -63 & Neutral & Disease \\
\hline rs759643470 & V9E & 0.74 & Tolerated & 61 & Effect & Disease \\
\hline rs765487840 & $\mathrm{H} 4 \mathrm{~L}$ & 0.03 & Damaging & -9 & Neutral & Disease \\
\hline rs775780092 & $\mathrm{H} 4 \mathrm{Y}$ & 0.11 & Damaging & -27 & Neutral & Disease \\
\hline
\end{tabular}

not find any reliable structural information for protein to carry out a FoldX stability analysis. Detailed results for selected 5 variants are supplied in Table 3.

3.2.6. Protein Stability Changes Found by I-Mutant 2.0. The prediction of stability changes of selected 5 nsSNPs by IMutant 2.0 is given in Table 3. The results are predicted to be either increase or decrease of the free energy change upon amino acid substitutions. Four out of five selective mutants were found to be decreased in protein stability and the remaining one mutant $(\mathrm{H} 341 \mathrm{Y})$ was predicted as increased in protein stability with reliability index (RI) 3 .

3.2.7. Visualization of Evolutionary Conserved Amino Acid Residues by ConSurf. ConSurf server is able to discriminate appropriately between the conservation caused by a short evolutionary time and genuine sequence conservation using Empirical Bayesian method. Our findings indicated that human $A D I P O R 1$ is highly conserved (Figure 2). The sequence alignment from different species revealed that residues A348 and $\mathrm{H} 341$ were located in highly conserved 
TABle 2: Refined SNPs obtained from PhD-SNP, PANTHER, SNPs\&GO, Fathmm, and PolyPhen-2 based classification systems.

\begin{tabular}{|c|c|c|c|c|c|}
\hline Amino acid change & PhD-SNP & PANTHER & SNPs\&GO & Fathmm & PolyPhen-2 \\
\hline G367R & Disease & Neutral & Neutral & Tolerated & Benign \\
\hline$A 348 G$ & Disease & Disease & Disease & Tolerated & Probably damaging \\
\hline$H 341 Y$ & Disease & Disease & Disease & Tolerated & Probably damaging \\
\hline$R 324 L$ & Disease & Disease & Disease & Tolerated & Probably damaging \\
\hline A307V & Disease & Neutral & Disease & Tolerated & Benign \\
\hline T296R & Disease & Neutral & Neutral & Tolerated & Benign \\
\hline V279G & Disease & Disease & Disease & Tolerated & Benign \\
\hline G275A & Neutral & Disease & Disease & Tolerated & Probably damaging \\
\hline V270M & Neutral & Disease & Neutral & Tolerated & Possibly damaging \\
\hline R264W & Disease & Disease & Disease & Tolerated & Possibly damaging \\
\hline $\mathrm{I} 251 \mathrm{~N}$ & Disease & Disease & Disease & Tolerated & Possibly damaging \\
\hline S231P & Disease & Neutral & Neutral & Tolerated & Possibly damaging \\
\hline$L 224 F$ & Disease & Neutral & Neutral & Tolerated & Probably damaging \\
\hline L251V & Disease & Neutral & Neutral & Tolerated & Probably damaging \\
\hline R202W & Disease & Disease & Disease & Tolerated & Benign \\
\hline F173L & Disease & Neutral & Neutral & Tolerated & Possibly damaging \\
\hline K170N & Neutral & Disease & Neutral & Tolerated & Probably damaging \\
\hline F145L & Disease & Neutral & Neutral & Tolerated & Benign \\
\hline$L 143 P$ & Disease & Disease & Disease & Tolerated & Probably damaging \\
\hline $\mathrm{R} 130 \mathrm{C}$ & Disease & Disease & Disease & Tolerated & Benign \\
\hline $\mathrm{R} 122 \mathrm{~W}$ & Disease & Disease & Neutral & Damaging & Benign \\
\hline M118K & Disease & Disease & Disease & Damaging & Benign \\
\hline P116S & Neutral & Disease & Neutral & Tolerated & Probably damaging \\
\hline D108N & Disease & Neutral & Neutral & Tolerated & Probably damaging \\
\hline $\mathrm{R} 91 \mathrm{H}$ & Disease & Neutral & Neutral & Tolerated & Benign \\
\hline E89D & Disease & Neutral & Disease & Tolerated & Benign \\
\hline E78K & Disease & Neutral & Neutral & Tolerated & Benign \\
\hline P70S & Neutral & Neutral & Neutral & Tolerated & Probably damaging \\
\hline $\mathrm{R} 40 \mathrm{P}$ & Disease & Unknown & Neutral & Tolerated & Benign \\
\hline G31E & Neutral & Unknown & Neutral & Tolerated & Probably damaging \\
\hline $\mathrm{A} 15 \mathrm{P}$ & Disease & Unknown & Neutral & Tolerated & Benign \\
\hline G14F & Disease & Unknown & Neutral & Tolerated & Benign \\
\hline $\mathrm{N} 13 \mathrm{~K}$ & Disease & Unknown & Neutral & Tolerated & Benign \\
\hline G12E & Disease & Unknown & Neutral & Tolerated & Benign \\
\hline V9E & Disease & Unknown & Neutral & Tolerated & Benign \\
\hline
\end{tabular}

regions and predicted to cause structural and functional impacts, respectively, on ADIPOR1 protein. On the other hand, the residues L224 and L143 had average conserved scores and the remaining one residue (R324) was located in conserved region of the protein.

3.3. Structural Analysis of Mutant Structures. The five predicted deleterious and disease causing variants were mapped to the PDB ID 3WXV native structure and substitution of amino acid residues was carried out using Swiss-PDB Viewer individually in order to generate five mutant modeled structures. After that, we calculated the total energy before and after energy minimization for both mutant model and wild type structures (Table 5). The values of total energy for five mutant modeled structures exhibit deviation from native structure considered before and after energy minimization. Five modeled structures (A348G, H341Y, R324L, L224F, and L143P) revealed an increase in energy (less favorable change) after energy minimization in comparing native structure. Among five screened mutations, H341Y showed the highest increase in energy which may be explained by the energetically unfavorable substitution of His to Tyr amino acids. 
TABLE 3: A list of selected variants for analyzing SNPeffect and I-Mutant tools.

\begin{tabular}{|c|c|c|c|c|c|c|}
\hline \multirow[b]{2}{*}{ Amino acid change } & \multirow[b]{2}{*}{$\begin{array}{c}\text { TANGO } \\
\text { Aggregation tendency } \\
\text { (dTANGO score) }\end{array}$} & \multicolumn{2}{|c|}{ SNPeffect } & \multirow[b]{2}{*}{$\begin{array}{c}\text { FoldX } \\
\text { Protein stability }\end{array}$} & \multicolumn{2}{|c|}{ I-Mutant } \\
\hline & & $\begin{array}{c}\text { WALTZ } \\
\text { Amyloid propensity } \\
\text { (dWALTZ score) }\end{array}$ & $\begin{array}{c}\text { LIMBO } \\
\text { Chaperone binding } \\
\text { tendency } \\
\text { (dLIMBO score) }\end{array}$ & & Prediction & RI \\
\hline A348G & $\begin{array}{c}\text { Not affected } \\
(-39.32)\end{array}$ & $\begin{array}{c}\text { Not affected } \\
(46.87)\end{array}$ & $\begin{array}{c}\text { Not affected } \\
(0.00)\end{array}$ & NP & Decrease & 8 \\
\hline H341Y & $\begin{array}{c}\text { Increased } \\
(222.09)\end{array}$ & $\begin{array}{c}\text { Decreased } \\
(-241.53)\end{array}$ & $\begin{array}{c}\text { Not affected } \\
(0.00)\end{array}$ & NP & Increase & 3 \\
\hline R324L & $\begin{array}{l}\text { Not affected } \\
(-1.01)\end{array}$ & $\begin{array}{c}\text { Not affected } \\
(0.49)\end{array}$ & $\begin{array}{c}\text { Not affected } \\
(0.00)\end{array}$ & NP & Decrease & 7 \\
\hline L224F & $\begin{array}{c}\text { Increased } \\
(51.62)\end{array}$ & $\begin{array}{c}\text { Not affected } \\
(-46.88)\end{array}$ & $\begin{array}{c}\text { Not affected } \\
(0.00)\end{array}$ & $\mathrm{NP}$ & Decrease & 8 \\
\hline L143P & $\begin{array}{l}\text { Decreased } \\
(-230.61)\end{array}$ & $\begin{array}{c}\text { Not affected } \\
(2.58)\end{array}$ & $\begin{array}{c}\text { Not affected } \\
(0.00)\end{array}$ & NP & Decrease & 4 \\
\hline
\end{tabular}

TABLE 4: Surface accessibility of wild type and mutants of ADIPOR1 protein.

\begin{tabular}{|c|c|c|c|c|}
\hline Amino acid change & Class assignment & $\begin{array}{c}\text { Relative surface } \\
\text { accessibility (RSA) }\end{array}$ & $\begin{array}{c}\text { Absolute surface } \\
\text { accessibility }\end{array}$ & $\begin{array}{c}Z \text {-fit score for RSA } \\
\text { prediction }\end{array}$ \\
\hline \multirow{2}{*}{$\mathrm{A} 348 G$} & Buried & 0.094 & 10.403 & -0.412 \\
\hline & Buried & 0.093 & 7.288 & -0.405 \\
\hline \multirow{2}{*}{ H341Y } & Buried & 0.070 & 12.769 & 0.848 \\
\hline & Buried & 0.063 & 13.463 & 0.712 \\
\hline \multirow{2}{*}{$\mathrm{R} 324 L$} & Exposed & 0.34 & 77.860 & -0.843 \\
\hline & Exposed & 0.352 & 64.470 & -0.734 \\
\hline \multirow{2}{*}{$\mathrm{L} 224 F$} & Buried & 0.029 & 5.273 & -0.077 \\
\hline & Buried & 0.027 & 5.519 & 0.018 \\
\hline \multirow{2}{*}{ L143P } & Exposed & 0.305 & 55.937 & -0.824 \\
\hline & Exposed & 0.329 & 46.628 & -0.942 \\
\hline
\end{tabular}

The zinc-binding domain is found in the intracellular layer of the membrane and zinc ion is coordinated by three His residues, His191, His337, and His341, of ADIPOR1 protein. In $\mathrm{H} 341 \mathrm{Y}$ variants, His is replaced by Tyr. Due to the presence of aromatic amino acid Tyr in 341 position of ADIPOR1 protein there may be a good chance to disrupt zinc coordination. Adiponectin stimulated AMPK phosphorylation and UCP2 upregulation are mediated by zinc-binding domain [46].

The results from TM score are delivered in Table 6. TM score was utilized in order to evaluate the topological similarity of two protein structures and RMSD measured the average distance between the backbones of two superimposed proteins [53]. The TM score for five variants reveals that structurally there are no differences between native and mutant modeled structures. It might be concluded that mutants and wild type structures are matched perfectly. We also considered another parameter (RMSD) in order to predict the structural similarity between native and mutant structures of ADIPOR1 protein. The higher is the RMSD value, the more is the deviation between the two structures which in turn fluctuates their functional activities. It can be seen from Table 6 that the RMSD values between the native structure and the mutant modeled structures are all similar. By considering the above two values of TM score and RMSD, it could be suggested that these mutations do not bring a significant alteration in the mutant structures with regard to the native protein structure.

Nonbonding interactions such as $\mathrm{H}$-bond has significant role in stabilizing the secondary structure of proteins [54]. Therefore, we have utilized the Swiss-PDB Viewer to visualize the hydrogen bonding pattern of five selected substituted amino acids with their surrounding amino acid residues in mutant proteins with regard to wild type (Figure 1). The hydrogen bonding pattern of variants A348G, L224F, and L143P has remained similar in comparison with wild type structure (PDB ID 3WXV). In variant H341Y, His341 indicates six hydrogen bonding interactions with Thr140, His141, Val344, Val345, His337, and Gln338, whereas mutant aromatic Tyr341 indicates five hydrogen bonding interactions. This has occurred due to the differences in the charge density and hydrophobicity between wild type and mutant residues. In variant $\mathrm{R} 324 \mathrm{~L}$, one $\mathrm{H}$-bond disappeared due to the substitution of Arg324 by Leu324. Additionally, A348G, H341Y, R324L, L224F, and L143P variants were analyzed for 


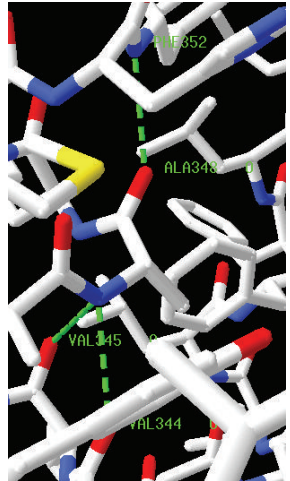

Wild type

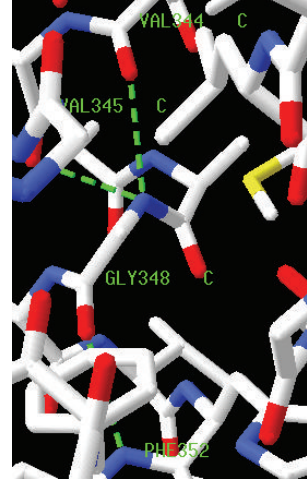

Variant_A348G

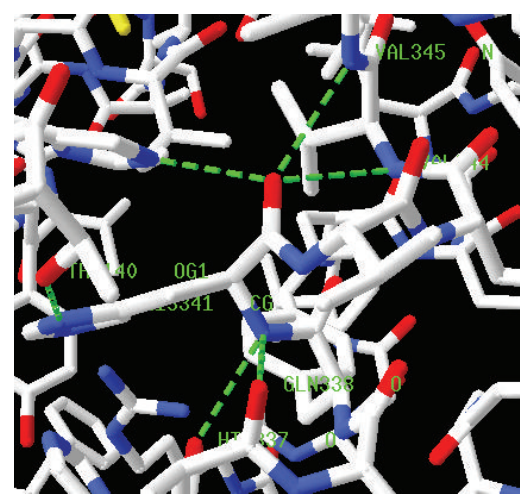

Wild type

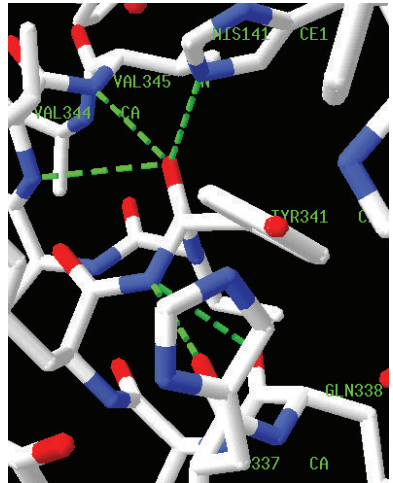

Variant_H341Y

(a)

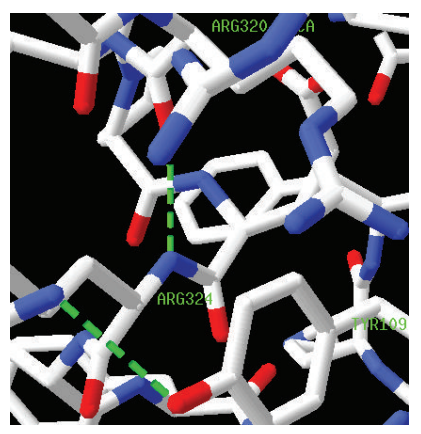

Wild type

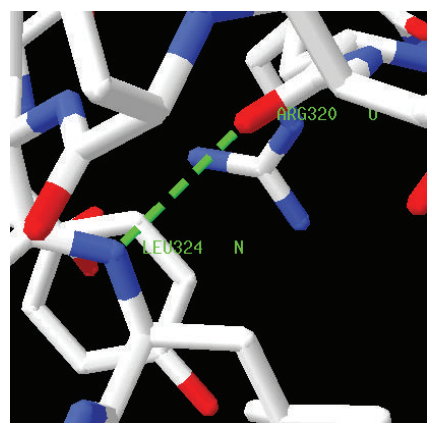

Variant_R324I

(c)

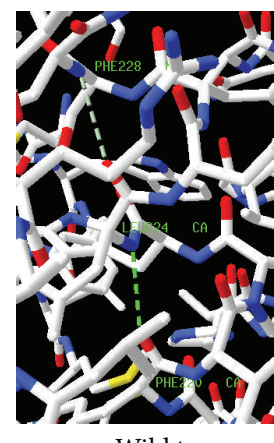

Wild type

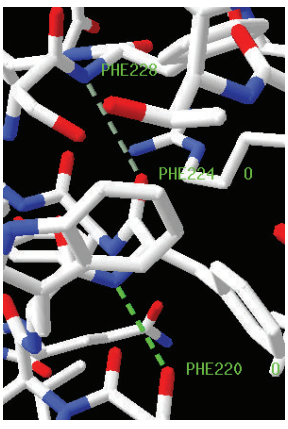

Variant_L224F

(d)

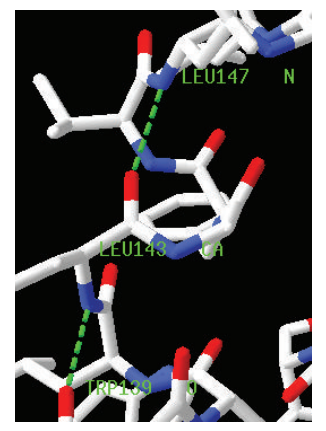

Wild type

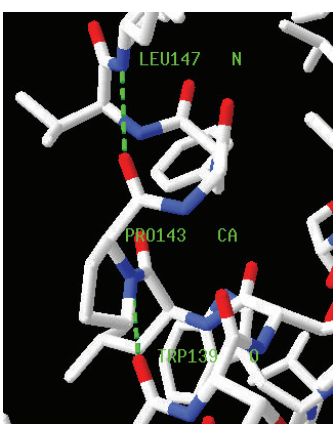

Variant_L143P

Figure 1: H-bond (green discontinuous line) and weak hydrogen bond (dark white discontinuous line) of wild type and mutant analogues with the adjacent amino acids residues. (a) At 348 position, three hydrogen bonds (H-bond) are observed with Val344, Val345, and Phe352 in both wild type (Ala348) and mutant (Gly348) structures. (b) His341 is visualized with six H-bonding interactions for Thr140, His141, His337, Gln338, Val344, and Val34, and one H-bond is abolished due to the replacement of mutant Tyr34 at the same position. (c) At 324 position, two H-bonds are observed with Tyr109 and Arg320 in native (Arg324) structure, but only one H-bond is found with Arg320 in mutant (Leu324) structure at the same position. (d) Phe228 is examined with single weak H-bonding in both native (Leu224) and substituted (Phe224) structures. In addition, single H-bond is also pictured with Phe220 in both structures. (e) Trp139 and Leu147 participated in forming two H-bonds at same position (143) in both mutant and wild type structures.

solvent accessibility and stability and significant changes in both parameters were seen for all five variants (Table 4).

\subsection{Analysis of Ligand Binding Sites and Protein-Protein Inter-} actions. FTSite identifies 3 ligand binding sites on ADIPOR1 protein (Figure 3). The amino acids found in these 3 sites of ADIPOR1 protein are given in Table 7. By the results of
FTSite, it is observed that our 5 selected variants are not involved among these sites.

STRING database predicted the functional interaction pattern of ADIPOR1 protein to other proteins in a cell. Strong functional associations of ADIPOR1 protein have been observed with $A D I P O Q, A P P L 1, L E P$, and INS partners (Figure 4). Besides, weak interactions with less confidence 


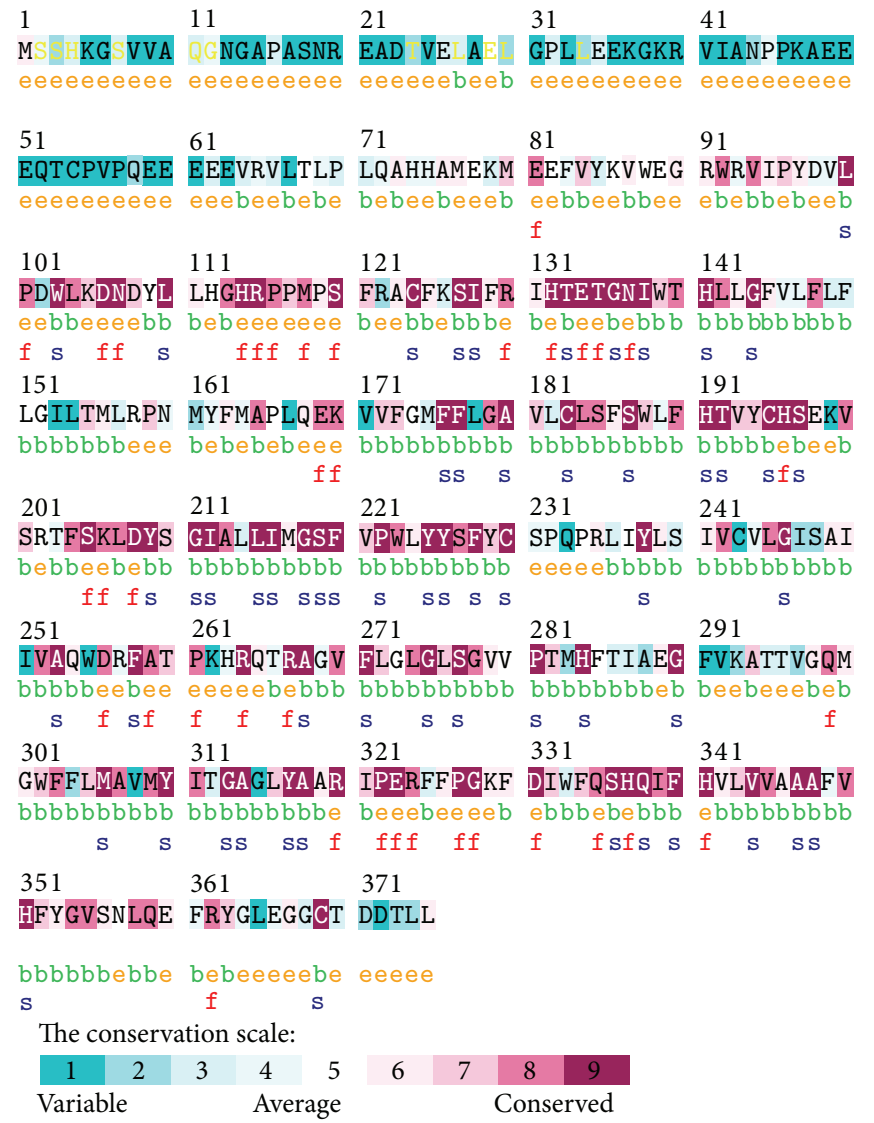

FIGURE 2: Unique and conserved amino acids in ADIPOR1 protein were predicted by ConSurf. Amino acids were ordered based on a conservation scale of 1-9 and highlighted as follows: blue residues (1-4) are variable, white residues (5) are average, and purple residues (6-9) are conserved. (e) Exposed residues are colored via an orange letter. (b) Buried residues are marked via a green letter. (f) Putative functional highly conserved and exposed residues are revealed with a red letter. (s) Predicted structural residues which are highly conserved and buried are indicated via blue letter.

TABLE 5: Total energy of native and mutant ADIPOR1 structures before and after energy minimization.

\begin{tabular}{lcc}
\hline Amino acid variants & Total energy before energy minimization $(\mathrm{kj} / \mathrm{mol})$ & Total energy after energy minimization $(\mathrm{kj} / \mathrm{mol})$ \\
\hline Native & -6555.888 & -11406.533 \\
A348G & -6468.591 & -11319.678 \\
H341Y & 86183.258 & -10520.564 \\
R324L & -6078.778 & -11107.321 \\
L224F & 372036.875 & -11199.636 \\
L143P & 179867.000 & -11088.141 \\
\hline
\end{tabular}

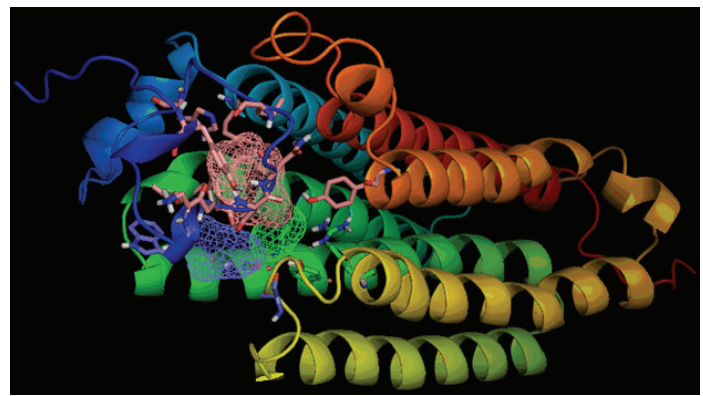

FIGURE 3: Binding of ligands in ADIPOR1 proteins ligand binding pocket 1-3 predicted by FTSite. No mutants were observed in binding site 1-3. 


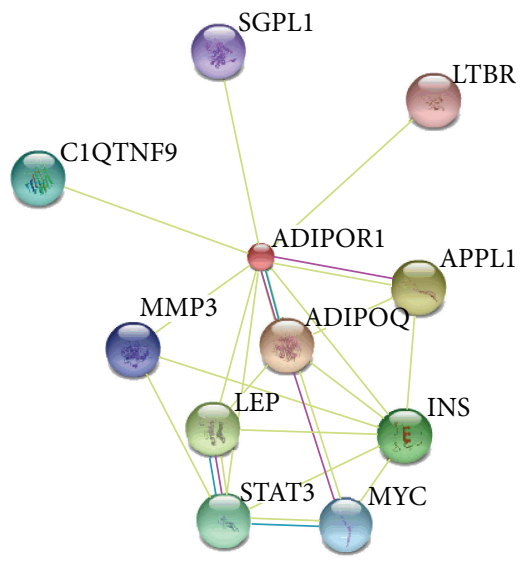

(a) Evidence view

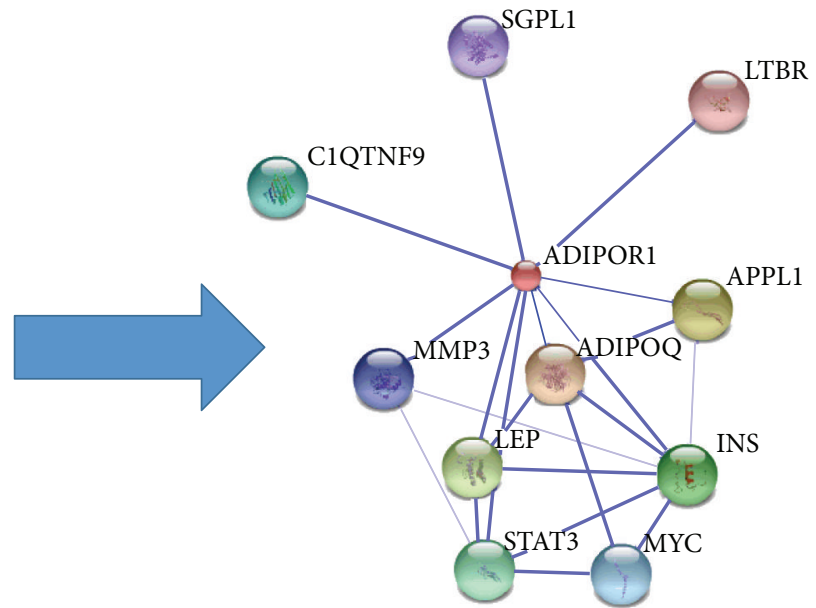

(b) Confidence view

ADIPOR1 functional partners:

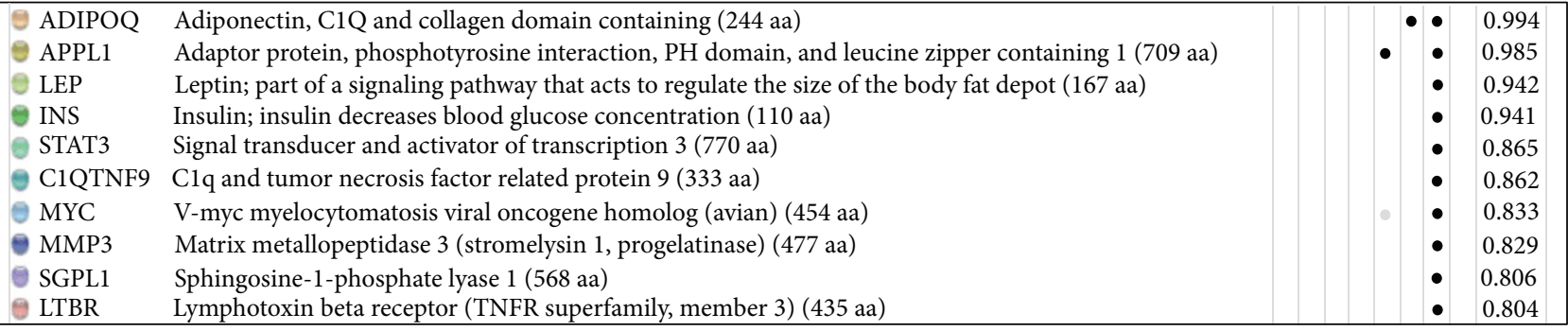

FIGURE 4: ADIPOR1 protein-protein interactions with 10 partners. One color is specified for each type of evidence in the predicted functional links (edges) among eight colored lines. (a) Only APPL1 with score of 0.985 is observed for interaction with ADIPOR1 by experimental and text-mining basis. From text-mining data, ADIPOR1 interactions are detected for ADIPOQ, APPL1, LEP, INS, STAT3, C1QTNF9, MYC, MMP3, $S G P L 1$, and $L T B R$ proteins with $0.994,0.985,0.942,0.941,0.865,0.862,0.833,0.828,0.806$, and 0.804 scores, respectively. (b) Strong association pattern (thick blue lines) of ADIPOR1 is predicted for ADIPOQ, APPL1, LEP, and INS partners with high confidence. The remaining partners have weak association and shown in the form of thin blue lines.

TABLE 6: RMSD value and TM score of mutant modeled structures of ADIPOR1 protein.

\begin{tabular}{lcc}
\hline Variants & RMSD value & TM score \\
\hline A348G & 0.00 & 1.00 \\
H341Y & 0.00 & 1.00 \\
R324L & 0.00 & 1.00 \\
L224F & 0.00 & 1.00 \\
L143P & 0.00 & 1.00 \\
\hline
\end{tabular}

have been observed for STAT3, C1QTNF9, MYC, MMP3, SGPL1, and LTBR proteins.

The associations between polymorphisms of ADIPOR1 gene (such as rs12733285 and rs1342387) and metabolic diseases such as diabetes, obesity, and insulin resistance have been reported [19, 20, 22, 23]. However, no such study has established the association between damaging nsSNPs (rs765425383, A348G; rs752071352, H341Y; rs759555652, R324L; rs200326086, L224F; and rs766267373, L143P) and diseases. Hence, the confirmation of these nsSNPs in any
TABLE 7: Residues at ligand binding sites of ADIPOR1 protein.

\begin{tabular}{lcc}
\hline Site 1 & Site 2 & Site 3 \\
\hline TYR A 97 & PHE A 190 & TRP A 103 \\
LEU A 104 & SER A 205 & LEU A 104 \\
LYS A 105 & ASP A 208 & ASP A 106 \\
ASP A 106 & TYR A 209 & PHE A 190 \\
ASN A 107 & ARG A 267 & TYR A 194 \\
LEU A 110 & TYR A 317 & SER A 201 \\
HIS A 114 & & SER A 205 \\
GLU A 134 & & ALA A 259 \\
PHE A 190 & & \\
HIS A 191 & & \\
TYR A 194 & & \\
TYR A 317 & & \\
\hline
\end{tabular}

disease is required to complement the existing limited body of knowledge. The combination of the analysis of human genetic variations of the ADIPOR1 gene together with the 
computational method to predict their possible functional impact may help in the analysis of ADIPOR1 gene variants and establish their effects on protein functional characteristics. Specifically, this approach permits the estimation of the probability of amino acid changes which can be detrimental for ADIPOR1 protein functions.

\section{Conclusion}

In this comprehensive computational study, we have identified five deleterious mutations (A348G, H341Y, R324L, L224F, and L143P) among the coding region of ADIPOR1 gene with the help of different bioinformatics tools. The variants were predicted to be similar to wild type ADIPOR1 protein structurally. However, decreased stability of mutant proteins has been observed with classical molecular dynamics study compared to wild type. Among the potential five nsSNPs, H341Y mutant has been found to cause considerable changes in amyloid forming propensity and aggregation tendency of ADIPOR1 protein. Additionally, there might be chance to disrupt the zinc coordination domain which is responsible for adiponectin stimulated MPK phosphorylation and UCP2 upregulation. The deleterious mutations of $A D I P O R 1$ should be further investigated to establish their roles in the pathogenesis of related diseases.

\section{Competing Interests}

The authors declare that there are no competing interests regarding this paper.

\section{Acknowledgments}

The authors would like to greatly acknowledge the technical support provided by the Department of Biochemistry and Molecular Biology, Jahangirnagar University, Savar, Dhaka, Bangladesh, during the study.

\section{References}

[1] J. M. Friedman, “Obesity in the new millennium," Nature, vol. 404, no. 6778, pp. 632-634, 2000.

[2] J. S. Flier, "Obesity wars: molecular progress confronts an expanding epidemic," Cell, vol. 116, no. 2, pp. 337-350, 2004.

[3] J. M. Olefsky and C. K. Glass, "Macrophages, inflammation, and insulin resistance," Annual Review of Physiology, vol. 72, no. 1, pp. 219-246, 2010.

[4] D. LeRoith and D. Accili, "Mechanisms of disease: using genetically altered mice to study concepts of type 2 diabetes," Nature Clinical Practice Endocrinology and Metabolism, vol. 4, no. 3, pp. 164-172, 2008.

[5] S. Gesta, Y.-H. Tseng, and C. R. Kahn, "Developmental origin of fat: tracking obesity to its source," Cell, vol. 131, no. 2, pp. 242256, 2007.

[6] J. Breitfeld, M. Stumvoll, and P. Kovacs, "Genetics of adiponectin," Biochimie, vol. 94, no. 10, pp. 2157-2163, 2012.

[7] J. Bełtowski, "Adiponectin and resistin-new hormones of white adipose tissue," Medical Science Monitor, vol. 9, no. 2, pp. RA55-RA61, 2003.
[8] J. Chen, B. Tan, E. Karteris et al., "Secretion of adiponectin by human placenta: differential modulation of adiponectin and its receptors by cytokines," Diabetologia, vol. 49, no. 6, pp. $1292-$ 1302, 2006.

[9] C. Hug and H. F. Lodish, "The role of the adipocyte hormone adiponectin in cardiovascular disease," Current Opinion in Pharmacology, vol. 5, no. 2, pp. 129-134, 2005.

[10] Y. Matsuzawa, "The metabolic syndrome and adipocytokines," FEBS Letters, vol. 580, no. 12, pp. 2917-2921, 2006.

[11] P. E. Scherer, "Adipose tissue: from lipid storage compartment to endocrine organ," Diabetes, vol. 55, no. 6, pp. 1537-1545, 2006.

[12] T. Kadowaki, T. Yamauchi, N. Kubota, K. Hara, K. Ueki, and $\mathrm{K}$. Tobe, "Adiponectin and adiponectin receptors in insulin resistance, diabetes, and the metabolic syndrome," Journal of Clinical Investigation, vol. 116, no. 7, pp. 1784-1792, 2006.

[13] T. Yamauchi, J. Kamon, Y. Minokoshi et al., "Adiponectin stimulates glucose utilization and fatty-acid oxidation by activating AMP-activated protein kinase," Nature Medicine, vol. 8, no. 11, pp. 1288-1295, 2002.

[14] E. Tomas, T.-S. Tsao, A. K. Saha et al., "Enhanced muscle fat oxidation and glucose transport by ACRP30 globular domain: acetyl-CoA carboxylase inhibition and AMP-activated protein kinase activation," Proceedings of the National Academy of Sciences of the United States of America, vol. 99, no. 25, pp. 16309-16313, 2002.

[15] X. Fang and G. Sweeney, "Mechanisms regulating energy metabolism by adiponectin in obesity and diabetes," Biochemical Society Transactions, vol. 34, no. 5, pp. 798-801, 2006.

[16] C. Hug, J. Wang, N. S. Ahmad, J. S. Bogan, T.-S. Tsao, and H. F. Lodish, "T-cadherin is a receptor for hexameric and highmolecular-weight forms of Acrp30/adiponectin," Proceedings of the National Academy of Sciences of the United States of America, vol. 101, no. 28, pp. 10308-10313, 2004.

[17] T. Yamauchi, J. Kamon, Y. Ito et al., "Cloning of adiponectin receptors that mediate antidiabetic metabolic effects," Nature, vol. 423, no. 6941, pp. 762-769, 2003.

[18] T. Yamauchi, Y. Nio, T. Maki et al., "Targeted disruption of AdipoR1 and AdipoR2 causes abrogation of adiponectin binding and metabolic actions," Nature Medicine, vol. 13, no. 3, pp. 332-339, 2007.

[19] K. J. Mather, C. A. Christophi, K. A. Jablonski et al., "Common variants in genes encoding adiponectin (ADIPOQ) and its receptors (ADIPOR1/2), adiponectin concentrations, and diabetes incidence in the Diabetes Prevention Program," Diabetic Medicine, vol. 29, no. 12, pp. 1579-1588, 2012.

[20] K. E. Peters, J. Beilby, G. Cadby et al., "A comprehensive investigation of variants in genes encoding adiponectin (ADIPOQ) and its receptors (ADIPOR1/R2), and their association with serum adiponectin, type 2 diabetes, insulin resistance and the metabolic syndrome," BMC Medical Genetics, vol. 14, article 15, 2013.

[21] V. Kaklamani, N. Yi, K. Zhang et al., "Polymorphisms of ADIPOQ and ADIPOR1 and prostate cancer risk," Metabolism: Clinical and Experimental, vol. 60, no. 9, pp. 1234-1243, 2011.

[22] N. A. Crimmins and L. J. Martin, "Polymorphisms in adiponectin receptor genes ADIPOR1 and ADIPOR2 and insulin resistance," Obesity Reviews, vol. 8, no. 5, pp. 419-423, 2007.

[23] N. Stefan, F. Machicao, H. Staiger et al., "Polymorphisms in the gene encoding adiponectin receptor 1 are associated with insulin resistance and high liver fat," Diabetologia, vol. 48, no. 11, pp. 2282-2291, 2005. 
[24] S. C. Collins, J. Luan, A. J. Thompson et al., "Adiponectin receptor genes: mutation screening in syndromes of insulin resistance and association studies for type 2 diabetes and metabolic traits in UK populations," Diabetologia, vol. 50, no. 3, pp. 555-562, 2007.

[25] M. Vaxillaire, A. Dechaume, V. Vasseur-Delannoy et al., "Genetic analysis of ADIPOR1 and ADIPOR2 candidate polymorphisms for type 2 diabetes in the Caucasian population," Diabetes, vol. 55, no. 3, pp. 856-861, 2006.

[26] S. Sunyaev, V. Ramensky, I. Koch, W. Lathe III, A. S. Kondrashov, and P. Bork, "Prediction of deleterious human alleles," Human Molecular Genetics, vol. 10, no. 6, pp. 591-597, 2001.

[27] Z. Wang and J. Moult, "SNPs, protein structure, and disease," Human Mutation, vol. 17, no. 4, pp. 263-270, 2001.

[28] R. Grantham, "Amino acid difference formula to help explain protein evolution," Science, vol. 185, no. 4154, pp. 862-864, 1974.

[29] D. Chasman and R. M. Adams, "Predicting the functional consequences of non-synonymous single nucleotide polymorphisms: structure-based assessment of amino acid variation," Journal of Molecular Biology, vol. 307, no. 2, pp. 683-706, 2001.

[30] C. Baynes, C. S. Healey, K. A. Pooley et al., "Common variants in the ATM, BRCA1, BRCA2, CHEK2 and TP53 cancer susceptibility genes are unlikely to increase breast cancer risk," Breast Cancer Research, vol. 9, no. 2, article R27, 2007.

[31] S. Paul, M. Solayman, M. Saha, and M. S. Hossain, "In silico analysis of the functional and structural impacts of nonsynonymous single nucleotide polymorphisms in the human paraxonase 1 gene," International Journal Bioautomation, vol. 19, no. 3, pp. 275-286, 2015.

[32] S. T. Sherry, M.-H. Ward, M. Kholodov et al., "DbSNP: the NCBI database of genetic variation," Nucleic Acids Research, vol. 29, no. 1, pp. 308-311, 2001.

[33] P. Kumar, S. Henikoff, and P. C. Ng, "Predicting the effects of coding non-synonymous variants on protein function using the SIFT algorithm," Nature Protocols, vol. 4, no. 7, pp. 1073-1082, 2009.

[34] L. Bao, M. Zhou, and Y. Cui, "nsSNPAnalyzer: identifying disease-associated nonsynonymous single nucleotide polymorphisms," Nucleic Acids Research, vol. 33, no. 2, pp. W480-W482, 2005.

[35] Y. Bromberg, G. Yachdav, and B. Rost, "SNAP predicts effect of mutations on protein function," Bioinformatics, vol. 24, no. 20, pp. 2397-2398, 2008.

[36] I. A. Adzhubei, S. Schmidt, L. Peshkin et al., "A method and server for predicting damaging missense mutations," Nature Methods, vol. 7, no. 4, pp. 248-249, 2010.

[37] R. Calabrese, E. Capriotti, P. Fariselli, P. L. Martelli, and R. Casadio, "Functional annotations improve the predictive score of human disease-related mutations in proteins," Human Mutation, vol. 30, no. 8, pp. 1237-1244, 2009.

[38] E. Capriotti, R. Calabrese, and R. Casadio, "Predicting the insurgence of human genetic diseases associated to single point protein mutations with support vector machines and evolutionary information," Bioinformatics, vol. 22, no. 22, pp. 27292734, 2006.

[39] D. J. Moore, L. Zhang, T. M. Dawson, and V. L. Dawson, "A missense mutation (L166P) in DJ-1, linked to familial Parkinson's disease, confers reduced protein stability and impairs homooligomerization," Journal of Neurochemistry, vol. 87, no. 6, pp. 1558-1567, 2003.
[40] H. A. Shihab, J. Gough, D. N. Cooper et al., "Predicting the functional, molecular, and phenotypic consequences of amino acid substitutions using hidden Markov models," Human Mutation, vol. 34, no. 1, pp. 57-65, 2013.

[41] G. De Baets, J. Van Durme, J. Reumers et al., "SNPeffect 4.0: online prediction of molecular and structural effects of proteincoding variants," Nucleic Acids Research, vol. 40, no. 1, pp. D935D939, 2012.

[42] E. Capriotti, P. Fariselli, and R. Casadio, "I-mutant2.0: predicting stability changes upon mutation from the protein sequence or structure," Nucleic Acids Research, vol. 33, no. 2, pp. W306W310, 2005.

[43] G. Celniker, G. Nimrod, H. Ashkenazy et al., "ConSurf: using evolutionary data to raise testable hypotheses about protein function," Israel Journal of Chemistry, vol. 53, no. 3-4, pp. 199206, 2013.

[44] B. Petersen, T. N. Petersen, P. Andersen, M. Nielsen, and C. Lundegaard, "A generic method for assignment of reliability scores applied to solvent accessibility predictions," BMC Structural Biology, vol. 9, no. 1, article 51, 2009.

[45] H. M. Berman, J. Westbrook, Z. Feng et al., "The protein data bank," Nucleic Acids Research, vol. 28, no. 1, pp. 235-242, 2000.

[46] H. Tanabe, Y. Fujii, M. Okada-Iwabu et al., "Crystal structures of the human adiponectin receptors," Nature, vol. 520, no. 7547, pp. 312-316, 2015.

[47] M. U. Johansson, V. Zoete, O. Michielin, and N. Guex, “Defining and searching for structural motifs using DeepView/SwissPdbViewer," BMC Bioinformatics, vol. 13, no. 1, article 173, 2012.

[48] Y. Zhang and J. Skolnick, "TM-align: a protein structure alignment algorithm based on the TM-score," Nucleic Acids Research, vol. 33, no. 7, pp. 2302-2309, 2005.

[49] C.-H. Ngan, D. R. Hall, B. Zerbe, L. E. Grove, D. Kozakov, and S. Vajda, "FTSite: high accuracy detection of ligand binding sites on unbound protein structures," Bioinformatics, vol. 28, no. 2, pp. 286-287, 2012.

[50] D. Szklarczyk, A. Franceschini, M. Kuhn et al., "The STRING database in 2011: functional interaction networks of proteins, globally integrated and scored," Nucleic Acids Research, vol. 39, no. 1, pp. D561-D568, 2011.

[51] R. J. Ellis, "Macromolecular crowding: obvious but underappreciated," Trends in Biochemical Sciences, vol. 26, no. 10, pp. 597604, 2001.

[52] C. M. Dobson, "The structural basis of protein folding and its links with human disease," Philosophical Transactions of the Royal Society of London, Series B: Biological Sciences, vol. 356, no. 1406, pp. 133-145, 2001.

[53] J. C. Jimenez-Lopez, E. W. Gachomo, M. J. Seufferheld, and S. O. Kotchoni, "The maize ALDH protein superfamily: linking structural features to functional specificities," BMC Structural Biology, vol. 10, article 43, 2010.

[54] J. A. Ippolito, R. S. Alexander, and D. W. Christianson, "Hydrogen bond stereochemistry in protein structure and function," Journal of Molecular Biology, vol. 215, no. 3, pp. 457-471, 1990. 

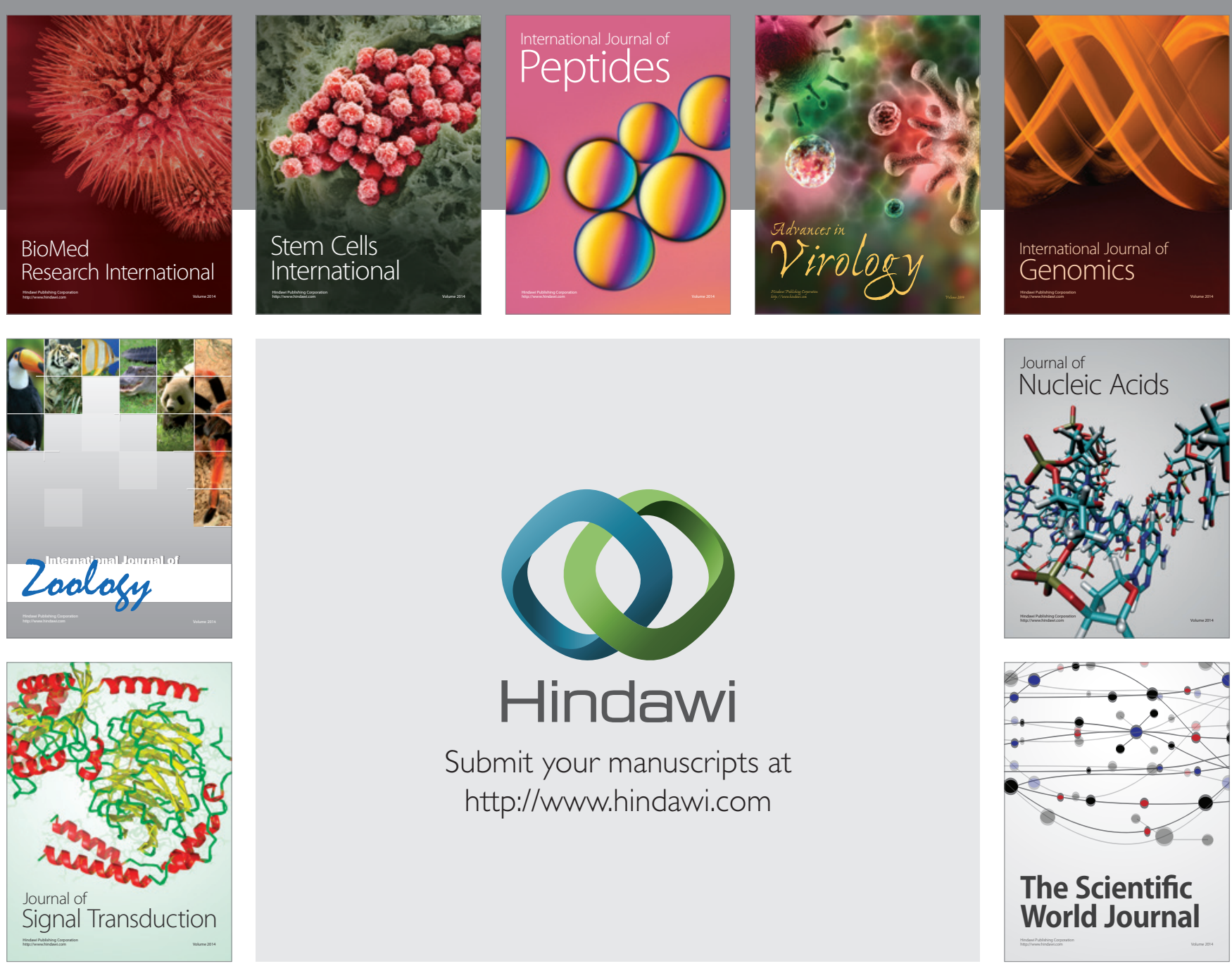

Submit your manuscripts at

http://www.hindawi.com
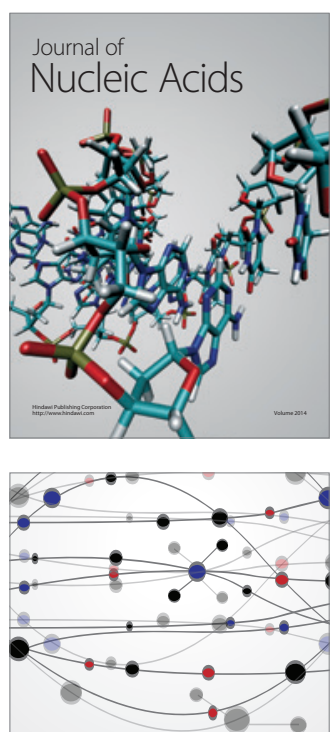

The Scientific World Journal
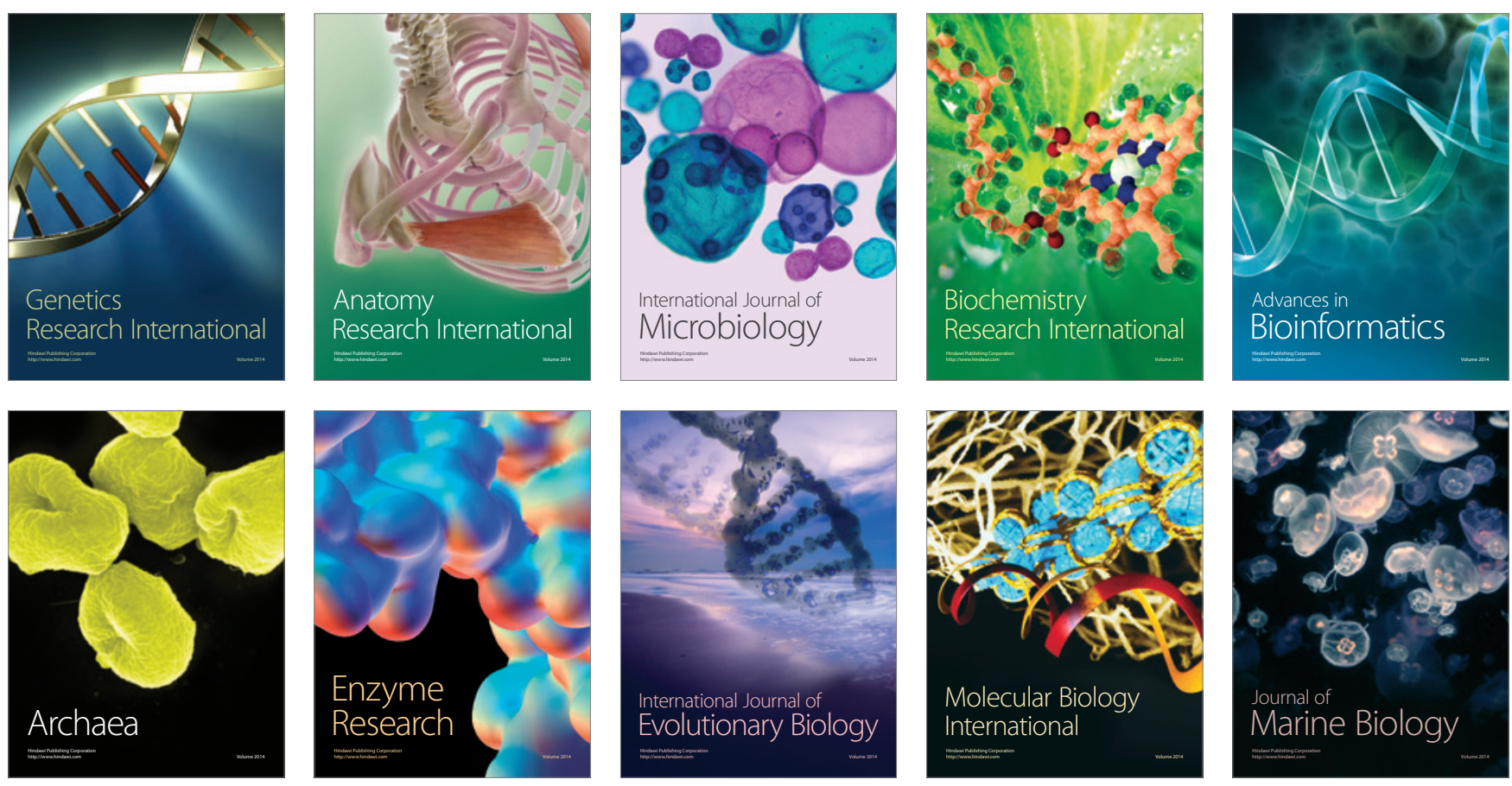